\title{
Diode-pumped 100-fs passively mode-locked Cr:LiSAF laser with an antiresonant Fabry-Perot saturable absorber
}

\author{
D. Kopf, K. J. Weingarten, L. R. Brovelli, M. Kamp, and U. Keller \\ Ultrafast Laser Physics, Institute of Quantum Electronics, Swiss Federal Institute of Technology, \\ ETH Hönggerberg HPT, CH-8093 Zürich, Switzerland
}

Received June 16, 1994

\begin{abstract}
We demonstrate a self-starting passively mode-locked diode-pumped Cr:LiSAF laser, achieving pulses as short as $98 \mathrm{fs}$ with an average power as high as $50 \mathrm{~mW}$. In continuous-wave operation, we demonstrate $140 \mathrm{~mW}$ of output power with a tunability of $100 \mathrm{~nm}$ FWHM. These results were achieved by use of lower $\mathrm{Cr}^{3+}$ doping (1.5\%), highbrightness diode lasers pumping from both sides of the crystal, and a low-loss antiresonant Fabry-Perot saturable absorber to start and sustain mode-locked operation.
\end{abstract}

Chromium-doped LiSrAlF$_{6}$ (Cr:LiSAF) is being widely investigated as a crystal for compact, tunable, low-cost mode-locked lasers with pulse widths below $100 \mathrm{fs}$ (Refs. 1 and 2) because of its large linewidth $(\approx 200 \mathrm{~nm})$ and its absorption band near $670 \mathrm{~nm}$, where diode laser pump sources are available. Recently, pulses as short as 220 fs were reported ${ }^{3}$ from such a system. However, obtaining short pulses with good average power is a challenge in a diodepumped Cr:LiSAF because of its low small-signal gain compared with that of other materials in diodepumped lasers such as Nd:YAG, Nd:YLF, or Nd:glass; the lower brightness of currently available laser diodes at $670 \mathrm{~nm}$ compared with that of laser diodes near $800 \mathrm{~nm}$; and the quenching of its upper-state lifetime at temperatures that exceed approximately $50^{\circ} \mathrm{C}$.

The characteristics described above explain why the first mode-locked results used diffraction-limited pumps, ${ }^{4,5}$ resulting in pulses as short as $33 \mathrm{fs}^{6}{ }^{6}$ The pump power required to demonstrate self-mode locking in these cases was comparable with powers available from diodes. However, the main constraint on longitudinal end pumping is mode matching, which means that the pump light must be focused to a size approximately equal to or less than the laser mode size in the crystal, over the pump absorption length. High-power diode lasers emit a beam with asymmetric, non-diffraction-limited properties. In the direction perpendicular to the junction, the beam is nearly diffraction limited, whereas in the other direction parallel to the junction, the beam is many times diffraction limited. In practice this means that the laser mode size inside the crystal is limited primarily by the pump beam quality in the direction parallel to the junction to achieve mode matching.

To obtain the highest small-signal gain, we wish to use the smallest laser mode size that we can mode match with our pump. By using a simple model, ${ }^{7}$ we can calculate the smallest spot size diameter $d_{\min }$ to which a diode of a given stripe size $l_{x}$ and divergence $\Theta_{x}$ can be mode matched over an absorption length $L \approx \alpha^{-1}$ of the crystal. We accomplish this by focusing the diode stripe into the middle of a cylinder of length $L$, using geometrically imaging and then choosing the magnification factor of the imaging system to minimize the size of the beam at the entrance to the cylinder (end of the crystal). The result is

$$
d_{\min }=\sqrt{L l_{x} \Theta_{x}} .
$$

Focusing to a smaller spot will increase the divergence and overfill the cylinder at either end. A larger spot will have less divergence but will overfill the cylinder along the entire length. Although this model is rather simple for typical diode-pumped cases, it will give us important insights into the scaling described in the next few paragraphs.

We also can estimate the temperature rise in the center of the crystal that is due to the absorbed pump power. Using a simple model of homogenous heat flow from a cylinder, we assume that the pump power is uniformly deposited within a cylinder of radius $r$ and length $L\left(\approx \alpha^{-1}\right)$. Assuming that the edge of the crystal of radius $R$ is held at a fixed temperature in a perfect heat sink, the temperature rise at the center of the pump is

$$
\Delta T=\frac{Q_{0}}{4 \pi k L}\left[1+2 \ln \left(\frac{R}{r}\right)\right],
$$

where $Q_{0}$ is the deposited heat and $k$ is the thermal conductivity. This temperature rise scales linearly with the absorbed pump power and inversely with the absorption length. Because of the logarithmic factor, changing the pump spot size of the crystal does not strongly affect the temperature rise. As the temperature approaches a critical value (the upper-state lifetime is reduced by one half at approximately $70^{\circ} \mathrm{C}$ in $\mathrm{Cr}: \mathrm{LiSAF}^{8}$ ), the small-signal gain significantly decreases. For a given pump power, therefore, there is a minimum absorption length (or maximum doping level) beyond which laser operation is no longer efficient, given a certain heat-sink temperature.

Given a pump diode with a large stripe size, Eq. (1) tells us that we can mode match by decreasing the absorption length, thereby allowing for tighter pump focusing and better mode matching. However, Eq. (2) 
tells us that the decreased absorption length will increasingly limit the maximum pump power because of upper-state lifetime quenching. The other option to improve laser performance is to decrease either the diode stripe length $l_{x}$ or its parallel divergence $\Theta_{x}$. The justification for our approach is the use of shorter-stripe diodes, which permits longer absorption length (lower-doped crystals) and higher pump power. Cooling the crystal will also permit stronger pumping, but in our experiments we cool the laser crystal only to temperatures readily achievable with thermoelectric coolers and avoid near-freezing or subfreezing temperatures, which would require isolating the crystal from humid air.

Figure 1 shows the setup of the laser cavity for both cw and mode-locked operation. In all experiments, the crystal heat-sink temperature was held at $\approx 10^{\circ} \mathrm{C}$ unless otherwise noted. Two diodes, with $400 \mathrm{~mW}$ of output power at $670 \mathrm{~nm}$ and emitting from 100 $\mu \mathrm{m}$ stripes with $6^{\circ}$ divergence parallel to the junction (Applied Optronics AOC-670-400), pump a 5-mm-long Brewster/Brewster $1.5 \% \mathrm{Cr}$ :LiSAF crystal from both sides. The pump beam is collected in its fast diverging direction by a $200-\mu \mathrm{m}$-diameter round cylindrical fiber lens with a refractive index of 1.8. The astigmatism introduced by the fiber lens is negligible, allowing the first round achromatic lens ( $f=200 \mathrm{~mm}$ ) to collimate both directions simultaneously. Then the beam is refocused through a cavity mirror by the second lens ( $f=120 \mathrm{~mm}$ ). Using a beam scan with a moving slit aperture, we measured the minimum spot size at the crystal position to be $80 \mu \mathrm{m}$ in diameter (10\% clip level), with a far-field full-angle divergence of $8^{\circ}(\approx 13$ times diffraction limited) in the direction parallel to the diode junction, and $60 \mu \mathrm{m}$ in the other direction, with a divergence of $2^{\circ}$. This provides mode matching to the $100 \mu \mathrm{m} \times 140 \mu \mathrm{m}$ diameter of the laser mode over the $\approx 1-\mathrm{mm}$ absorption length of the crystal, approximately following Eq. (1).

We investigated the effect of upper-state lifetime quenching on laser efficiency by tuning the crystal heat sink temperature up to $50^{\circ} \mathrm{C}$, with two different $\mathrm{Cr}^{3+}$ doping levels and three output couplers (Fig. 2). With $3 \% \mathrm{Cr}$ :LiSAF (absorption length $L \approx 0.5 \mathrm{~mm}$ ), the output power decreased at lower temperatures than for the lower $1.5 \%$ doping ( $L \approx 1 \mathrm{~mm}$ ). For an ideal four-level laser, increasing the laser's total losses (i.e., increasing output coupling) normally causes the laser threshold to increase proportionally. However, in Cr:LiSAF this extra pump power deposits more heat, increasing the crystal temperature and decreasing the upper-state lifetime and the small-signal gain. Therefore reaching threshold requires more pump power, resulting in reduced laser output power. This shows the significance of keeping the intracavity losses as low as possible to prevent thermal upper-state lifetime quenching from dramatically reducing the laser's efficiency.

Using the $1.5 \%$ doped crystal and both diodes, we obtained $140 \mathrm{~mW}$ of output power with a $2 \%$ output coupler in the cw cavity $(580 \mathrm{~mW}$ of absorbed pump power and a laser threshold of $160 \mathrm{~mW}$ ). After inserting a birefringent plate, we obtained approximately $100 \mathrm{~mW}$ of average power, tunable over a range of $100 \mathrm{~nm}$ FWHM [Fig. 3(a)], limited by our cavity mirrors. The linewidth was measured to be less than $0.1 \mathrm{~nm}$, the resolution limit of our optical spectrum analyzer.

To mode lock the laser, we chose to use an antiresonant Fabry-Perot saturable absorber (A-FPSA), which has successfully demonstrated selfstarting mode locking in several Nd-doped lasers. ${ }^{9-11}$ For this experiment, we fabricated a structure for $\approx 830 \mathrm{~nm}$ as shown in the inset of Fig. 1. The saturable absorber consists of a low-temperaturegrown $\left(T=350^{\circ} \mathrm{C}\right)$ multiple-quantum well layer with a peak in the excitonic absorption at $830 \mathrm{~nm}$, embedded between the two mirrors. The lower mirror is molecular-beam-epitaxy grown, and the top mirror is evaporated. The A-FPSA, operating at antiresonance at $830 \mathrm{~nm}$, has a free spectral range of more than $100 \mathrm{~nm}$. However, the overall range of this specific A-FPSA is limited to $\sim 50 \mathrm{~nm}$ by the bandwidth of the bottom mirror. We measured a bitemporal reflectivity response with a slow recovery time of $16 \mathrm{ps}$ owing to recombination and a fast recovery time of 150-200 fs owing to intraband thermalization of the carriers. ${ }^{12}$ The slow recovery time provides sufficient modulation for the longer pulses in the start-up phase, and the faster recovery time contributes to the femtosecond pulse formation. We consider the A-FPSA instead of Kerr-lens mode locking to be the dominating effect, because the laser was operated in the middle of the cavity stability regime, not close to the cavity stability limit as is usually the case in achieving significant Kerr-lens mode locking.

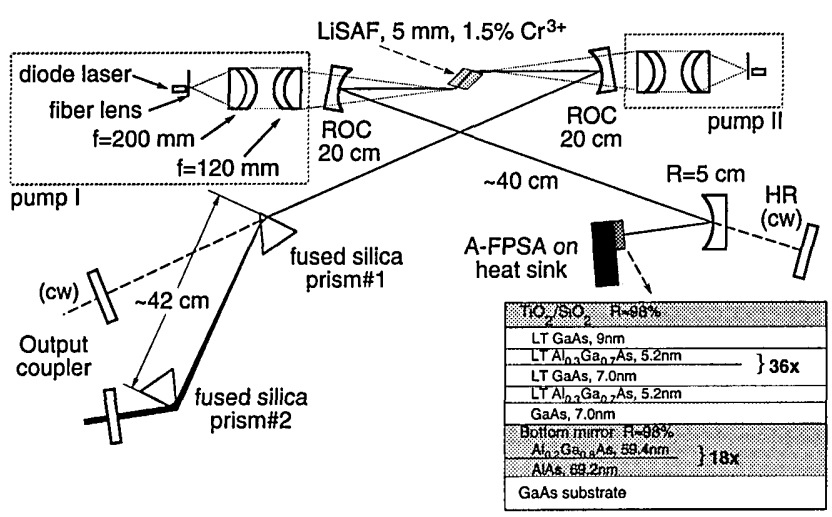

Fig. 1. Schematic of the laser cavity. HR, high reflector; $\mathrm{ROC}$, radius of curvature.

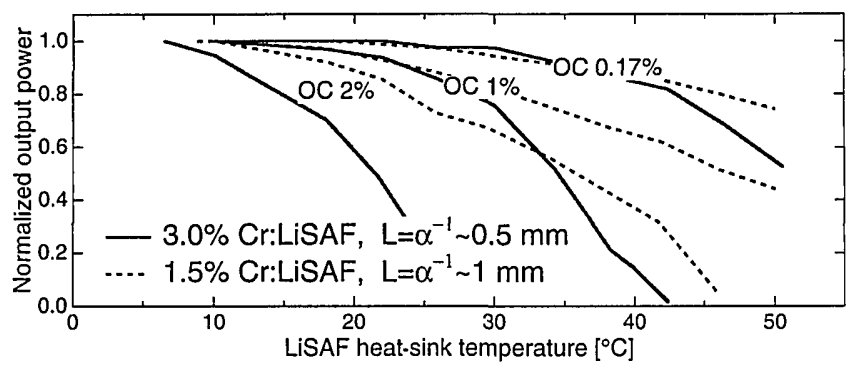

Fig. 2. Normalized output power versus crystal heat-sink temperature for two different crystal doping levels $(1.5 \%$ and $3 \%)$ and three different output couplers (OC's) $(0.17 \%, 1 \%$, and $2 \%)$. The absorbed pump power was kept constant at $300 \mathrm{~mW}$. 


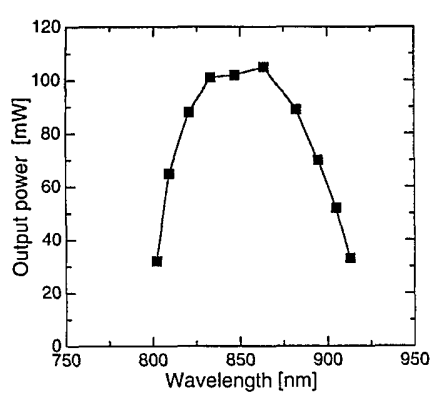

(a)

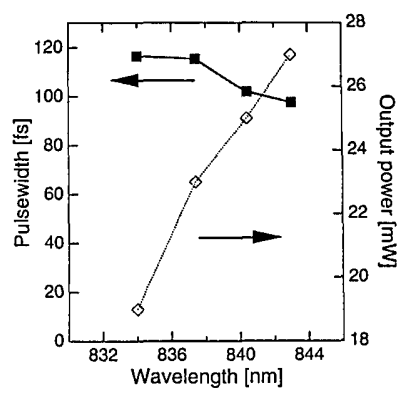

(b)
Fig. 3. (a) $\mathrm{Cw}$ tunability over $100 \mathrm{~nm}$ FWHM with a birefringent plate, (b) mode-locked tunability of approximately $10 \mathrm{~nm}$. The wavelength was tuned with a knife edge inserted into the laser beam between the two prisms.

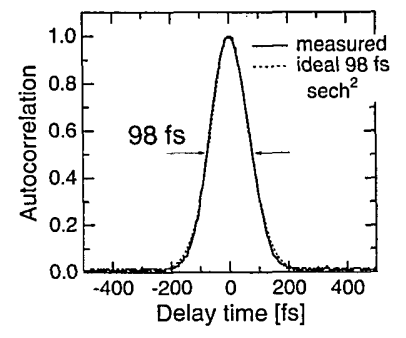

(a)

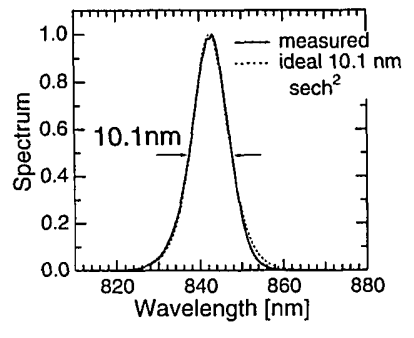

(b)
Fig. 4. (a) Mode-locked pulse width with a noncollinear autocorrelation, (b) the corresponding optical spectrum.

In addition, the intensity-dependent nonlinearity is much smaller, because of the larger laser mode size inside the laser crystal required by diode-pumping, than in typical Kerr-lens mode-locking lasers, such as Ti:sapphire lasers.

To achieve mode-locked operation, we reconfigured the cavity as shown in Fig. 1 by inserting dispersion compensation prisms in one arm, replacing one end mirror with a curved mirror that focuses the laser beam onto the A-FPSA with a $15-\mu \mathrm{m}$ radius, and reducing the output coupler to $1 \%$. Self-starting mode locking was obtained with sub-100-fs pulses of $27-\mathrm{mW}$ average output power (Fig. 4) at a repetition rate of $120 \mathrm{MHz} .{ }^{13}$ The optical spectrum was $10 \mathrm{~nm}$ wide, giving a time-bandwidth product of 0.42 . We measured mode-locked wavelength tunability over a range of $10 \mathrm{~nm}$ by inserting a knife edge into the laser beam between the two prisms [Fig. 3(b)]. The system exhibits good long-term stability, with the output power and pulse width remaining constant for hours.

Using an A-FPSA with reduced insertion losses (1\% instead of $2 \%$ ), we increased the output coupling to $2 \%$ while keeping the total losses and therefore the intracavity power the same. This resulted in approximately twice as much average output power than before, as we would expect, and again we measured near-transform-limited 100 -fs pulses with an average output power of $50 \mathrm{~mW}$.

The use of the A-FPSA structure, compared with the use of an antireflection-coated multiple-quantum- well saturable absorber, ${ }^{3}$ was critical for reducing the insertion loss of the device because, as was seen in the cw measurements, we pay an extra penalty in terms of laser efficiency for all added loss to the laser because of the thermal quenching of the upper-state lifetime. One limitation of our current devices is tunability. However, tunability of greater than $100 \mathrm{~nm}$ has been demonstrated ${ }^{14}$ with similar multiple-qunatum-well saturable absorbers in a coupled-cavity mode-locked Ti:sapphire laser. With proper design of the saturable absorber and the lower molecular-beam-epitaxy-grown mirror, we expect that comparable tunability also should be possible. Finally, although a Kerr-lens mode-locked diode-pumped Cr:LiSAF laser should be possible, features of the A-FPSA are its ability to provide enough saturation for self-starting of the laser even at the lower laser powers available with diode-pumping and to allow the cavity to be designed in the center of its stability range, where we can achieve the most robust performance.

The authors thank Lightwave Electronics for providing the fiber lenses. This work was supported by the Swiss National Fund.

\section{References}

1. P. M. W. French, R. Mellish, and J. R. Taylor, Opt. Lett. 18, 1934 (1993).

2. F. Balembois, P. Georges, and A. Brun, Opt. Lett. 18, 1730 (1993).

3. P. M. Mellish, P. M. W. French, J. R. Taylor, P. J. Delfyett, and L. T. Florez, Electron. Lett. 30, 223 (1994).

4. A. Miller, P. LiKamWa, B. H. T. Chai, and E. W. Van Stryland, Opt. Lett. 17, 195 (1992).

5. J. M. Evans, D. E. Spence, W. Sibbett, B. H. T. Chai, and A. Miller, Opt. Lett. 17, 1447 (1992).

6. N. H. Rizvi P. M. W. French, and J. R. Taylor, Opt. Lett. 17, 1605 (1992).

7. T. Y. Fan and A. Sanchez, IEEE J. Quantum Electron. 26, 311 (1990).

8. M. Stalder, M. Bass, and B. H. T. Chai, J. Opt. Soc. Am. B 9, 2271 (1992).

9. U. Keller, D. A. B. Miller, G. D. Boyd, T. H. Chiu, J. F. Ferguson, and M. T. Asom, Opt. Lett. 17, 505 (1992).

10. U. Keller, T. H. Chiu, and J. F. Ferguson, Opt. Lett. 18, 217 (1993).

11. U. Keller, Appl. Phys. B 58, 347 (1994).

12. L. R. Brovelli, U. Keller, and T. H. Chiu, "Design and operation of antiresonant Fabry-Perot saturable semiconductor absorbers for mode-locked solid-state lasers," J. Opt. Soc. Am. B (to be published).

13. D. Kopf, K. J. Weingarten, L. Brovelli, M. Kamp, and U. Keller, in Conference on Lasers and Electro-Optics, Vol. 8 of 1994 OSA Technical Digest Series (Optical Society of America, Washinton, D.C., 1994), paper CPD22.

14. G. R. Jacobovitz-Veselka, U. Keller, and M. T. Asom, Opt. Lett. 17, 1791 (1992). 